\begin{tabular}{|l|l|} 
REVISTA Revista Educación \\
ISSN: 0379-7082 \\
ISSN: 2215-2644 \\
revedu@gmail.com \\
Universidad de Costa Rica \\
Costa Rica
\end{tabular}

\title{
Educación Superior y formación mercantilizada: ¿existe alternativa?
}

Trujillo Vargas, José Jesús

Educación Superior y formación mercantilizada: ¿existe alternativa?

Revista Educación, vol. 44, núm. 1, 2020

Universidad de Costa Rica, Costa Rica

Disponible en: http://www.redalyc.org/articulo.oa?id=44060092004

DOI: https://doi.org/10.15517/revedu.v44i1.34234

Esta obra está bajo una Licencia Creative Commons Atribución-NoComercial-SinDerivar 3.0 Internacional. 


\title{
Educación Superior y formación mercantilizada: ¿existe alternativa?
}

\author{
José Jesús Trujillo Vargas \\ Escuela Internacional de Mediación (EIM), España \\ trujillo215@hotmail.com \\ (D) http://orcid.org/0000-0002-5384-670X
}

Higher Education and Commodification of Education: Is There an Alternative?

DOI: https://doi.org/10.15517/revedu.v44i1.34234

Redalyc: http://www.redalyc.org/articulo.oa?id=44060092004

Recepción: 23 Marzo 2019

Aprobación: 15 Julio 2019

\section{Resumen:}

En un mundo donde todo parece relativo, todo es cambiante y nada permanece inalterable, nos encontramos un contexto educativo-formativo-universal actual, donde se nos vende la existencia de una única manera de proceder para poder adquirir una determinada formación solvente acorde al futuro desempeño laboral, al que esta conducirá a quienes opten, o se les permita, acceder a esta. La retórica de los discursos neoliberales posee las suficientes trampas como para convencer a todos aquellos miembros de la comunidad educativo-formativa que anhelan un futuro laboral estable o a aquellos que, siendo parte activa de dicha formación (profesores/as), intentan cumplir los requisitos imperantes con el fin último de la mejora de su desempeño laboral, aunque en dicho intento elaboren acciones que van en contra de la propia esencia de lo que significa educar y/o formar. Este trabajo centra su atención en reflexionar, a raíz de una revisión bibliográfica sobre la temática, sobre los condicionantes ético-sociales insertos en este sistema formativo y las consecuencias que él mismo está teniendo no ya para el profesorado universitario en sí, sino para los futuros docentes que se están formando en la actualidad. Las conclusiones más importantes de este estudio giran en torno a analizar las consecuencias académico-formativas y la incidencia en las formas que se producen los conocimientos científicos. E, igualmente, se incide en las consecuencias sociales, económicas, formativas... que este tipo de formación traerá consigo para los educandos de estos futuros profesores/as citados/as y para el conjunto de la sociedad en general y si existe alternativa a dicho modelo.

Palabras Clave: Educar, Formar, Neoliberalismo, Mercado laboral, Profesorado universitario.

\section{Abstract:}

In a world where everything seems relative and changeable, leaving nothing unaltered, our current context of higher education/ university learning faces a situation where it is sold on only one way of doing things to ensure success. The rhetoric of neoliberal discourses has enough traps to convince active members of the educational-training community who yearn for future job stability to fulfill prevailing requirements in order to improve their performance although it may include actions that defy traditional education or training. This study comprises a bibliographic review on the ethical-social conditions embodied in teacher training and the consequences for the university teaching staff and future teachers who are currently being trained. Significant conclusions drawn from this study include educational-training analysis and impact on attainment of scientific knowledge as well as social, economic and educational consequences for future teachers and society if there are no alternatives to this model.

KEYWORDS: Educate, Train, Neoliberalism, Labor Market, University Instructors.

\section{INTRODUCCIÓN}

El presente trabajo pretende incitar a la reflexión al conjunto de la comunidad universitaria en aras de un replanteamiento sobre su quehacer cotidiano y cómo este se encuentra condicionado por las políticas y prácticas neoliberales que ponen el énfasis en el producto, más que en el proceso y en la creación del valor comercial, más que en la generación de un conocimiento que propicie el bien común.

El neoliberalismo conforma tanto una ideología como un conjunto de prácticas, eminentemente económicas y políticas, que está marcando el devenir de las sociedades contemporáneas desde la década de los 80 del siglo pasado y redefiniendo la nueva versión del capitalismo en la era de la globalización. A diferencia de la visión liberal, en que el mercado se entiende por oposición al Estado, en el paradigma neoliberal el objetivo 
es introducir la lógica del mercado -la competencia- en todos los ámbitos sociales; no solo en el económico o productivo, sino en la educación, la cultura, los servicios públicos o la propia experiencia vital (Aibar, 2014).

Para el neoliberalismo, la competencia es la característica fundamental de las relaciones sociales. Afirma que el mercado produce beneficios que no se podrían conseguir mediante la planificación, y convierte a los ciudadanos en consumidores cuyas opciones democráticas se reducen, como mucho, a comprar y vender, proceso que supuestamente premia el mérito y castiga la ineficacia. Por tanto, la desigualdad es una virtud: una recompensa al esfuerzo y un generador de riqueza que beneficia a todos. La pretensión de crear una sociedad más equitativa es contraproducente y moralmente corrosiva. El mercado se asegura de que todos reciban lo que merecen. (Monbiot, 2016)

El individualismo exacerbado que promueve este paradigma, fundamentado en ideas tales como: si quieres, puedes, el poder lo tienes tú, tienes que demostrar que la mediocridad es para otros o la única salida es la excelencia, ha conseguido deconstruir el imaginario social en favor de lo eficiente, de lo productivo, de lo rentable... Restando importancia a todo lo que no dependa del individuo como ser autosuficiente y menospreciando a este como agente activo de cambio, capaz de movilizarse en favor de la lucha de aquellos colectivos que sufren situaciones de injusticia social: por discriminación étnica, sexual, de género, etc., llegando el propio individuo a la autopercepción de que son causas pérdidas por las cuales poco o nada se puede hacer, con la firme idea de que esto solo puede pasarle a quien no hace todo lo posible por cambiar su situación (asumiendo que nunca lo sufrirá en carnes propias). Percepción que suele modificar cuando el sistema lo excluye y degrada. ¿Quién puede asegurar hoy día, por poner un ejemplo clarividente, que su situación laboral es estable y no peligra su puesto o dicha situación no se convierte en cada vez en más precaria?

En los regímenes neoliberales, en los que la preeminencia de la forma-empresa ha colonizado los modos de comprensión de la vida social y de los sujetos, los dispositivos de poder han intensificado determinadas tecnologías gubernamentales, fundamentalmente todo aquello que puede ser vinculado con los procesos de regulación de las subjetividades y cuya naturaleza no sea directamente imperativa o autoritaria (Amigot y Martínez, 2013, p. 105)

El discurso sutil de los mecanismos de este sistema, posibilita el aceptar sus preceptos. Como ejemplo de lo descrito se tiene los medios de comunicación.

Que el bien común sea abordado mediante procesos que en realidad benefician a grupos concretos es posible porque: a) por un lado, no se trata de conservar características del orden social sino, prioritariamente y como eslogan permanente, de cambiar, emprender, movilizar, innovar, como si la innovación fuera positiva en sí misma y se desdibujaran los efectos desiguales que tiene; b) porque la lógica neoliberal desplaza a la responsabilidad individual el peso de las limitaciones que se ejercen a nivel colectivo; por ejemplo, todo el mundo es un emprendedor en potencia, aunque algunos prefieran la pasividad y el inmovilismo -egoísta-, al esfuerzo moralizado como la verdadera aportación a la sociedad; y c) porque las transformaciones están dictadas por la necesidad. El cambio es una realidad y una necesidad social: por tanto, es deseable adelantarse a él y facilitarlo (Boltanski, 2008, citado por Amigot y Martínez, 2013).

Si lo extrapolamos al sistema educativo, en sus diferentes niveles, podemos decir que este se presenta como propicio para hacer que esta imposición neoliberal se naturalice como algo positivo: exigencia por la excelencia, competitividad, posibilidad de conseguir lo que se quiera si existe esfuerzo..., a fin de cuentas, el ideal a seguir para mejorar. Hasta el punto de que hablar hoy día de competencias o estándares de evaluación, por citar dos ejemplos, se ha convertido en algo rutinario e interiorizado por los diferentes miembros de la comunidad educativa, como si siempre hubieran estado ahí. Y, en muchos casos, sin analizar el trasfondo mercantilista que implica; no ya en el discurso, sino lo que supone su puesta en práctica en relación con la igualdad de oportunidades del alumnado y/o con la justicia curricular. A lo que ha ayudado, sin duda, un fenómeno de desvinculación-despolitización acaecido a lo largo y ancho del siglo XX (Rivero, 2013).

La afirmación de que la competencia universal depende de un proceso de cuantificación y comparación universales es otra de las paradojas del neoliberalismo. Provoca que los trabajadores, las personas que buscan empleo y los propios servicios públicos 
se vean sometidos a un régimen opresivo de evaluación y seguimiento, pensado para identificar a los triunfadores y castigar a los perdedores (Monbiot, 2016, p. 2)

En el ámbito universitario, esto se ve reflejado no solo en la precariedad laboral: más horas de clases, profesores/as contratados/as a tiempo parcial que ejecutan el mismo número de horas que quienes tienen plaza fija recibiendo un sueldo netamente inferior, imposición de un perfil docente-investigador con clara intención de una, execrable, práctica de mercantilización del conocimiento, sino, muy especialmente, en la lucha encarnizada por acreditar programas, trabajo investigativo, formación a todos los niveles... Lo que no solo homogeneiza los procesos, sino que los convierte en meros productos eficientes a los que todos (instituciones y personas) han de llegar, si quieren seguir subsistiendo. De esta forma, los/las profesores/as universitarios/as se han convertido en los nuevos oprimidos, cuya máxima aspiración pasa por demostrar que son competentes en un sistema que ha sido engendrado para que el individualismo y la cuantificación se conviertan en las únicas varas de medir del triunfo personal, social y profesional.

Como génesis de esta problemática, conviene hacer hincapié en que la universidad ha sido siempre una entidad productora de la cultura hegemónica, y por tanto, un ente de reproducción social del sistema imperante (Aibar, 2018). El matiz a resaltar es que en la actualidad el potencial de creación de saber, conocimiento y cultura de las universidades está siendo atrapado por los intereses empresariales (Galcerán, 2010a) lo que degenera en que la institución favorezca una mayor desigualdad de oportunidades y una más que evidente falta de democracia. Muestra de lo que decimos, son los llamados paper, por los que se dictaminan, en gran medida, otorgar o no la acreditación como investigador/a. Sin embargo, no cualquier artículo científico es considerado de calidad por la Agencia de Evaluación del profesorado universitario, pues si estos no están publicados en revistas indexadas en JCR [Journal Citacion Reports] no serán tenidos tan en cuenta o desmerecerán el reconocimiento de la comunidad científica actual. La gestión de JCR pertenece a Thomson Reuters, empresas que antes de su fusión tenían como fuente de ingresos, entre otros, la explotación de los yacimientos de gas y petróleo del Mar del Norte y en proveer información a los mercados financieros, respectivamente (Reig, 2014).

El mundo de la publicación académica está también fuertemente privatizado. Unas pocas empresas multinacionales (Elsevier, Springer, Wiley-Blackwell, Taylor \& Francis y Sage) controlan más de la mitad de revistas científicas en todo el mundo -hasta hace pocas décadas en manos de sociedades científicas- y obtienen márgenes de beneficio que rondan el $40 \%$. Su modelo de negocio se basa en no remunerar el trabajo de las autoras y revisoras (investigadoras todas ellas) y en cobrar subscripciones, cada vez más caras, a las instituciones académicas en que trabajan. Esta lucrativa industria está incluso utilizando la actual demanda de Open Access para obtener aún más ingresos (Aibar, 2018).

Todo esto conforma el llamado capitalismo cognitivo, o aquel abierto a diversas líneas de desarrollo, donde las fuerzas neoliberales intentan encauzar únicamente en su beneficio las nuevas formas productivas emergentes, en especial aquellas que giran en torno al trabajo intelectual, inmaterial y/o cognitivo (Galcerán, 2010b).

Este texto tiene por objeto desentrañar las verdaderas intenciones neoliberales que se esconden bajo los discursos: de la enseñanza por competencias, de los rankings categorizadores de la investigación docente, de la excelencia del profesorado o de la propia evaluación estandarizada, como medio para promover una alternativa de resistencia a nivel sociopedagógico.

La metodología para desarrollar este trabajo ha estado fundamentada en la búsqueda y el análisis, durante los dos últimos años, de varios artículos publicados en la última década, a través de una búsqueda pormenorizada tanto en bases de datos: Dialnet, Redalyc, Scielo, Google Scholar, como en libros y artículos de periódicos afines a la temática. Aquellos artículos que no presentaban una vinculación intrínseca entre neoliberalismo y sistema formativo-educativo fueron desechados en una primera o segunda revisión/ clasificación. Evidentemente, el trabajo también se ha fundamentado en una serie de obras científicas clásicas 
que permiten una cosmovisión analítica cronológica que, si cabe, da mayor sustento científico a nuestras argumentaciones.

\section{PEDAGOGÍA OPRESORA MERCANTILISTA}

La sinergia entre universidad y empresa no consiste en un encuentro entre dos instituciones diferenciadas, sino en la supeditación de la dinámica universitaria al objetivo económico de rentabilizar los conocimientos adquiridos, vendiéndolos a los potenciales interesados y privilegiando los intereses de las empresas activas en los campos respectivos (Galcerán, 2010a, p. 16).

El imaginario social creado sobre la importancia de estar formados para poder adquirir un puesto de trabajo en un futuro no muy lejano, se agudiza de manera encarnizada en tiempos donde el poder adquisitivo mayoritario reside en unas cuantas y privilegiadas manos. De tal forma que los estudiantes universitarios tienden a endeudarse (cuando no les queda más remedio) a fin de poder adquirir los conocimientos necesarios que les posibiliten acceder a un puesto de trabajo solvente. Aunque los discursos que alientan este tipo de círculo formativo-económico, se suelen olvidar (intencionadamente) de que el mercado de trabajo es fluctuante y depende de muchos factores ajenos a la voluntad de quien lo pone todo en juego para poder aspirar a una estabilidad socioeconómica personal futura.

Es evidente que la educación debe tener conexión con el mercado de trabajo; lo cuestionable es que solo en función de ello se establezcan sus metas y se evalúe su impacto, convirtiéndose así el proceso educativo en una labor meramente técnica. Negando el eminente carácter social y ético del acto educativo que implica conflictos de intereses, valores e ideales. Esta mutilación no admite que la comunidad educativa participe activa y democráticamente en el proceso educativo (Díez, 2010).

Desde hace un tiempo a esta parte, hemos podido comprobar, desde los diferentes estamentos del sistema educativo-formativo, como los planes de estudio, las programaciones de aula y las guías didácticas, se han reorientado hacia una formación por y para la adquisición de competencias. Bajo el paraguas de sus postulados: saber, saber hacer y saber ser, la educación por competencias ha generado un nuevo paradigma totalizador que trata de finiquitar cualquier debate en torno a cómo, qué y cuándo enseñar y evaluar. A partir de ahora el alumnado que adquiera unas determinadas competencias, no solo superará una determinada asignatura, sino que también asegurará, con ello, poder desenvolverse de manera eficiente en su vida personal y profesional. Aunque para ello tenga que olvidar o renunciar a sus creencias, principios o ideología en aras de consolidar, a nivel socioeconómico, su presente y su futuro. El sometimiento a la voluntad del empresariado, y, por ende, a la adaptabilidad, pase lo que pase, al trabajo, son los valores añadidos que no aparecen descritos en ningún programa educativo de cualquier entidad que esté inmersa en el sistema (ya sea de primaria, de secundaria o universitaria).

No resulta en absoluto evidente que esas competencias formen parte de una educación centrada en el desarrollo de las capacidades de los estudiantes que tienda a aumentar su aptitud para operar con los conocimientos adquiridos. Más bien se trata de construir una subjetividad autoprogramable y flexible que oscile entre la creatividad y la sumisión (Galcerán, 2010b, p. 105)

Por otra parte, nunca será del todo comprobable en la práctica la adquisición o no de las competencias por parte de los alumnos/as (de cualquier nivel), porque el escenario de enseñanza no es idéntico, en ningún caso, a los múltiples escenarios donde dichos alumnos interactúan y donde tienen que poner a prueba la interiorización de dichas competencias.

En el marco de la Educación Superior, se ha posibilitado una mutación tanto de las funciones de los formadores como en las relaciones que establecen entre ellos y con su ámbito de actuación. Del clásico profesor/a que debía poseer una noción amplia de su área de conocimiento, lo cual no era óbice para dedicar tiempo al ocio en su vida personal: viajar, ir al teatro, leer por placer... hemos pasado a profesores/as que deben 
saber de todo, e incluso en su tiempo de ocio deben estar pendientes de lo que ocurre en su trabajo, pues la carga lectiva e investigativa (publicaciones, congresos, correcciones, preparación de clases...) es tal que no se les permite tener muchos descuidos personales. De esta guisa, una herramienta como Internet se ha convertido, en muchos casos, en un medio al servicio de ese profesor/a esclavo/a que tiene que seguir demostrándole al sistema que es apto. Así pues, podemos decir que las tecnologías móviles encajan perfectamente con las disposiciones y el habitus psíquico del sujeto académico neoliberal: comprobar, supervisar, descargar (Gill, 2015).

El neoliberalismo encontró un terreno fértil en los académicos, cuya predisposición a «trabajar duro»y «hacerlo bien» encajan perfectamente con las exigencias neoliberales de contar con sujetos autónomos, automotivados y responsables. La ausencia de resistencia a la penetración del neoliberalismo en las universidades es en parte el resultado de estas prácticas individualizadoras y atomizadoras, de los silencios alrededor de ellas, también del hecho de que la gente está demasiado agotada para resistir y además no sabe a qué resistir o cómo hacerlo (Gill, 2015, p. 56).

Más sangrante, si cabe, resulta el modus operandi para la categorización del profesorado investigador universitario. Las revistas cientificas (cuyas publicaciones componen uno de los factores más importantes en la categorización del profesorado) entran en una lucha encarnizada por ascender en los rankings de impacto, con el aliciente de tener mayor visibilidad y reconocimiento. Consecuentemente, los profesores/ as-investigadores/as universitarios entran en el juego de querer publicar cuanto más mejor en las revistas mejor posicionadas. Aunque el impacto de la investigación que ejecuten, no sirva (en muchos casos) para evidenciar hallazgos que realmente supongan un verdadero impacto a nivel político-socio-educativo. Además del prestigio, entre los colegas, que supone el hecho de publicar en revistas de reconocimiento mundial, existe un factor determinante que anima al profesorado a entrar en el juego de este mercantilismo de ideas, no es otro que una mayor retribución salarial si consiguen una categorización relevante como investigador. De esta forma, los nuevos excluidos, serán aquellos que se aparten, o no compartan, de los objetivos que se presentan, desde las diversas instancias de poder, como los únicos posibles para convertirse en verdaderos investigadores.

Agencias de calidad como la ANECA (dirigida a acreditar la calidad del profesorado) y la CNEAI (que reconoce, a través de los sexenios, la actividad investigadora desarrollada en períodos de cinco años), priorizan como criterios de valor la publicación de artículos en revistas científicas incluidas en bases de datos elaboradas por dos empresas privadas, Thomsom Reuters y Elsevier (propietarias respectivamente de las bases de datos WoS y Scopus), en detrimento de otros formatos y modos de canalizar la actividad investigadora. Estos criterios de valor reproducen una lógica colonial, son un efecto de lógicas de poder geopolíticas (que marginan y desprecian las revistas científicas no anglosajonas, que imponen sin discusión el inglés como lengua neutra) y, a pesar de las críticas de todo tipo que han generado, conservan intacta su capacidad de definir quién vale y quién no. (Indocentia, 2016 citado por Fernández, 2016)

Igualmente, más allá del factor salarial, tener tramos de investigación reconocidos y acreditados por las diferentes agencias de evaluación, se ha convertido en requisito para el acceso a los cuerpos docentes universitarios (tanto en universidades públicas como en privadas), para la dirección de tesis doctorales o para participar como miembros de los tribunales de tesis (Gómez y Jódar, 2013).

Este ciclo recurrente se cierra, y se retroalimenta continuamente, gracias a la estandarización concienzuda, sesgada e interesada de los procesos formativos: desde la evaluación y clasificación del profesorado, hasta la medición de todas y cada una de las acciones que elabora el profesorado en su aula (de manera más o menos implícita), pasando por las evaluaciones de los programas de formación, con el afán de conseguir los registros de calidad y excelencia, tan cacareados en nuestros tiempos por la comunidad científico-académica.

Las pruebas estandarizadas conforman la interrelación y legitimación de tres juegos de saber-poder que son inseparables: relaciones de saber; poder disciplinario y normalizador; y biopoder de regulación. La legitimación de las relaciones de saber se perpetúa por medio de los conocimientos específicos que son medidos en las evaluaciones estandarizadas que se encargan de formar un tipo de individuo predeterminado. La medición de dichos saberes responde a un poder disciplinario y normalizador que se ejerce directamente 
en la producción de sujetos dóciles y categorizados en las líneas divisorias de la normalidad y anormalidad. El biopoder de regulación se extiende a nivel global por medio de mediciones estandarizadas que introduce a los sujetos en conjuntos poblacionales, como elemento regulador de estas relaciones de saber y poder (Saura y Luengo, 2015).

La legitimación de los procesos de estandarización del rendimiento, aunque no únicamente, tiene mucho que ver con la perpetuación del economicismo como poder de dominación de los sistemas educativos. De igual forma que PISA es considerada como medida internacional comparativa, en exclusiva, del capital humano de las regiones, utilizada como indicador del potencial de competitividad económica mundial (Rizvi y Lingard, 2009), JCR y Scopus, se han convertido en las exclusivas varas de medir de la capacidad investigativa docente, en detrimento no solo de otro tipo de baremos, sino del propio ejercicio docente. Toda vez que la importancia de la docencia es ínfima, en relación a la investigación publicada a través de papers para las agencias evaluadoras de la calidad docente (que suelen tener, a su vez, a estas bases de datos como gurús a las que apostarlo todo).

\section{3. ¿EXISTEN POSIBILIDADES REALES DE UNA RESISTENCIA PEDAGÓGICA?}

El gran problema radica en cómo podrán los oprimidos, como seres duales, inauténticos, que alojan al opresor en sí, participar de la elaboración de la pedagogía para su liberación. Solo en la medida en que descubran que alojan al opresor podrán contribuir a la construcción de su pedagogía liberadora (Freire, 2003, p. 34).

La salida de esta situación enclaustrada no resulta en modo alguno sencilla, en la medida en que el propio sistema se ha encargado de sellar las posibles fisuras creadas por las desavenencias que pudieran surgir en torno a la situación existente, descrita hasta este punto.

Conviene significar que todos (o casi todos) hemos sido formados a través de una concepción bancaria de la educación (Freire, 2003), donde cualquier alternativa, que como alumnos propusiéramos a lo establecido, era menospreciada, subyugada y desestimada en aras de que el statu quo siguiera prevaleciendo. Lo que se hace mucho más evidente, si cabe, en el sistema educativo-formativo mercantilizado, que venimos analizando, aunque de una manera más sutil, pues el propio profesorado se muestra convencido, en muchas ocasiones, de la bondad pedagógica de dicho sistema. Lo mismo ocurre con gran parte del alumnado, que no posee las herramientas críticas necesarias como para cuestionar la calidad de la formación que recibe, ni el sistema que propugna la mencionada formación por competencias.

Segmentar los diferentes sectores de la comunidad educativa es un objetivo, implícito, que el sistema formativo neoliberal ha conseguido con creces. El individualismo, aislamiento, recrudecimiento de las exigencias profesionales (y por tanto la exacerbada competitividad entre profesores) y exceso de laboriosidad, en aras de cumplir con los cánones académicos exigidos, ha traído consigo la casi inexistente resistencia por parte del profesorado.

En muchos casos el miedo a perder el puesto de trabajo, pese a la inestabilidad económica y profesional, es otra de las principales razones (como así ocurre en la mayoría de sectores sociales) para que no exista un levantamiento, por parte del profesorado, que posibilite un verdadero cambio, hacia unas condiciones de mayor dignidad personal y profesional. También influye de manera patente la escasa alternativa laboral existente en el mercado actual, mucho más restringida y devaluada para el ámbito socioeducativo/formativo que, para otros ámbitos, más relacionado con lo productivo/mercantilista. De tal guisa y a tenor de lo comentado, son voces minoritarias las que llevan a cabo publicaciones u otras acciones de denuncia hacia este despropósito educativo-formativo.

Uno de los aspectos fundamentales para que reaccionemos no es otro que analizar todos los factores descritos para darnos cuenta de que nos encontramos, posiblemente, en el momento histórico de mayor deterioro de la autonomía investigativo-pedagógica docente. De tal manera que podemos afirmar, con las evidencias hasta aquí analizadas, que nuestra docencia está secuestrada. 
La dimensión ético-humanística-social, consustancial a toda institución formativo-educativa, debe hacer que cuestionemos continuamente este modelo mercantilista, ayudando a construir una alternativa pedagógico-político-social que parta de la no sumisión a los legados neoliberales, apostando por una verdadera universalización del conocimiento en contra de la globalización interesada, manipulada y sesgada de las élites precursoras de este capitalismo cognitivo, que apuesta por la generación de ciudadanos dependientes y sumisos a los legados de dichas élites.

En la dinámica de terapia organizacional que constituye la responsabilización social universitaria, no es posible escapar de una reflexión sobre el significado social, ético y político de la formación universitaria, la producción de conocimientos científicos y el rol político de la ciencia en el mundo actual. Esta reflexión entra en el rubro de lo que nosotros llamamos 'impactos cognitivos y epistemológicos', al lado de los otros tres tipos de impactos universitarios (impactos organizacionales hacia dentro, incluyendo tanto la dimensión laboral como la medioambiental; impactos formativos hacia los estudiantes; e impactos sociales hacia todos los agentes externos con los cuales se vincula la universidad) (Vallaeys, 2014, p. 112)

Si verdaderamente nos dedicamos a esta profesión por la pasión intrínseca de enseñar y por el firme convencimiento de que otra realidad social más justa y equitativa es posible (aunque este sistema va desposeyendo a esta profesión de su rasgo vocacional), convendría aunar esfuerzos para deconstruir estos valores deshumanizadores, que nos alejan de lo verdaderamente educativo, a través de acciones de resistencia conjunta para el no sometimiento a las leyes mercantiles. Sería fundamental construir espacios de encuentro que eviten el silenciamiento de nuestros derechos como trabajadores de la enseñanza. Donde se combata el miedo a las represalias y se construya una cultura de resistencia que haga proclive el necesario y continuo debate sobre qué estamos haciendo y para qué.

La producción de otros espacios de pensamiento y resistencia pasa por generar otras formas de reconocimiento colectivo a nuestro trabajo, distintas a las que nos ofrece la empresa Thomsom Reuters; por dar valor a vínculos en los que no solo haya instrumentalización del otro; por no someter la investigación a los circuitos rentables; por intentar construir espacios comunes a pesar de nuestra fragmentación y jerarquización; por no desertar de la docencia y proteger ese espacio de relación (Indocentia, 2016 citado por Fernández, 2016, p. 2.).

Esto nos obliga, necesariamente, a que estos espacios de encuentro estén abiertos a toda la comunidad educativa, en aras de una construcción de una alternativa pedagógico-social desde abajo. Para ello, hay que comenzar por combatir, de manera decidida e imperiosa, el ego que hemos ido adquiriendo con base en la remuneración social o laboral que hayamos obtenido a tenor de lo que este sistema premia o rechaza. "La formación requiere dudar, desidentificarse, desaprender, perder, soltar lo sobrante, quitar capas, eliminar, evaporar, etc., para ser menos ego y más conciencia. A veces los conocimientos previos son el mayor obstáculo para formarse" (Herrán, 2012, p.182).

A modo de ejemplo (en ningún caso de recetario), convendría construir espacios que ayuden a combatir los males del profesorado universitario, del que nos habla Barnés (2014). De tal manera que:

- Se construyan relaciones profesionales donde la jerarquía no sea más importante que el trabajo con un fin común y construido desde el juicio crítico y democrático, combatiendo las prácticas feudalistas que se vienen dando desde tiempos inmemoriales en la institución universitaria. Para ello convendría construir una cultura de convivencia y fomentar acciones firmes y radicales para erradicar, por ejemplo, el mobbing entre compañeros profesores/as.

- Mostremos una actitud de insumisión ante el papel burocrático que nos ha sido asignado, tendiendo a dedicar más horas a la preparación de las clases o a una verdadera investigación que nazca de las necesidades comunitarias, que a rellenar documentos estandarizados que en muchas ocasiones, más que favorecer el proceso, solo sirven para maniatar a un profesorado cuya profesión se sigue desvirtuando constantemente y desde diferentes frentes.

- Se favorezca una lucha unida y cooperativa en contra del desprestigio que azota a la profesión docente (mucho más acentuado en tiempos de crisis socioeconómicas). 
- Se fomente la lucha y reivindicación por la creación de criterios que aboguen por la transparencia a la hora de la consecución de plazas o del acceso, por ejemplo, a becas de investigación.

- Se lleven a cabo líneas de presión en favor de unas condiciones sociolaborales acordes a la función docente, favoreciéndose con ello menores diferencias salariales entre profesores/as titulares y aquellos que no lo son.

\section{Conclusiones}

Los estudios universitarios se configuran, así como un conjunto de servicios cognitivos, ofrecidos en diferentes puntos del planeta para formar una fuerza de trabajo especializada y sofisticada, capaz de aprovechar los mejores talentos del mundo, especialmente sensible a la demanda de capas con ciertos recursos de cualquier parte. La promoción de la lengua inglesa como idioma universal refuerza esa tendencia y permite que ciertas universidades, colocadas en la cúspide de la jerarquía, gocen de una posición de dominio a escala mundial, atrayendo estudiosos de cualquier parte del mundo (Galcerán, 2010a, p. 20)

Esta mercantilización globalizada del conocimiento va acompañada de la expropiación de conocimientos a las personas y de la limitación de su valor de uso (Galcerán, 2010a), siendo considerados únicamente conocimientos fundamentales aquellos que favorecen códigos de conducta dictados por las empresas a las que estos futuros profesionales van a pertenecer.

La colonización de la investigación científica por parte de intereses privados está siendo promovida y potenciada por políticas científicas neoliberales que interpretan la relevancia social de la ciencia como simple transferencia al sector empresarial privado. Se trata de políticas científicas que incentivan el uso de patentes para favorecer la comercialización del conocimiento, que, simultáneamente, promocionan los sistemas de evaluación de la investigación puramente cuantitativos, promoviendo de forma explícita la competitividad por encima de la colaboración (colaborar para competir mejor nos dicen) y que, mediante las llamadas politicas de la excelencia, concentran cada vez más los recursos en unos pocos investigadores y centros de investigación, depauperando a pasos agigantados el resto del sistema universitario público (Mirowski, 2011; Aibar, 2018).

El giro neoliberal en la ciencia contemporánea está teniendo consecuencias muy importantes, no solo en la organización y la gestión de la ciencia y las instituciones académicas, sino en las formas en que se producen los conocimientos científicos: en el tipo de resultados que se generan, en los métodos que se consideran más legítimos o en los enfoques teóricos más secundados (Aibar, 2014).

Si admitimos que en nuestras sociedades capitalistas la dinámica que hemos descrito conduce a la servidumbre de las conciencias; si reconocemos como un hecho que el pobre absolutiza frecuentemente su ignorancia en provecho del patrón y de los que son como el patrón, los cuales se convierten en los jueces y fiadores de todo el saber, se impone la conclusión de que la tarea más inmediata es, para el pedagogo, el rescate puro y simple de estos hombres, la liberación de sus conciencias y el descubrir su propia personalidad, por encima de sus alienaciones (Silva, 1976). El caso es que en el ámbito académico en el cual nos desenvolvemos, solemos pensar que no hay patrón o que este no influye de manera decisiva en nuestro quehacer cotidiano.

A través de este documento hemos tratado de reflexionar sobre la posibilidad de llevar a cabo otro tipo de acciones educativo/formativas con un mayor componente humanístico y ético. Lo que no es óbice para considerar que existe un presente y futuro nada halagüeño, a nivel pedagógico formativo, en virtud de todo lo comentado en este trabajo.

\section{REFERENCIAS BIBLIOGRÁFICAS}

Aibar, E. (2014). Ciència oberta, encerclament digital i producció colllaborativa. En T. Iribarren, O. Gassol y E. Aibar (Eds.), Cultura i tecnologia: els reptes de la producció cultural en l'era digital (pp. 99-120). Lleida: Punctum.

Aibar, E. (2018). La transformación neoliberal de la ciencia: El caso de las Humanidades Digitales. ArtefaCToS, 7(1), 13-28. Recuperado de: http://www.rebelion.org/docs/241006.pdf 
Amigot, P. y Martínez, L. (2013). Gubernamentalidad neoliberal, subjetividad y transformación de la universidad. La evaluación del profesorado como técnica de normalización. Athenea Digital, 13(1), 99-120.

Barnés, H. (2014). Trabajos tóxicos: Los 8 males del profesor universitario. Recuperado de: https://bit.ly/1j7YJ6P

Díez, E. J. (2010). La globalización y sus repercusiones en educación. Revista Electrónica Interuniversitaria de Formación del Profesorado, 13 (2), 23-39.

Fernández, A. (19 de febrero, 2016). Disciplinar la investigación, devaluar la docencia: cuando la universidad se vuelve empresa. Entrevista al colectivo de profesores y estudiantes Indocentia sobre la transformación neoliberal de la Universidad. El diario. Recuperado de: https://bit.ly/1QPVBXJ

Freire, P. (2003). Pedagogía del oprimido. Madrid: Siglo XXI.

Galcerán, M. (2010a). La educación universitaria en el centro del conflicto. En Edu-Factory y Universidad Nómada (Comps.), La universidad en conflicto. Capturas y fugas en el mercado del saber (pp. 13-39). Madrid: Traficantes de sueños.

Galcerán, M. (2010b). La mercantilización de la universidad. REIFOP, 13 (2), 89-106. Recuperado de: http://www .redalyc.org/pdf/2170/217014950008.pdf

Gómez, L. y Jódar, F. (2013). Ética y política en la universidad española: la evaluación de la investigación como tecnología de la subjetividad. Athenea Digital, 13(1), 81-98.

Gill, R. (2015). Rompiendo el silencio. Las heridas ocultas de la universidad neoliberal. Arxius, 32, 45-58.

Herrán, A. (2012). Enfoque radical e inclusivo de la formación. REICE. Revista Iberoamericana sobre Calidad, Eficacia y Cambio en Educación, 12(2), 163-264.

Mirowski, P. (2011). Science-Mart. Privatizing American Science. Harvard: Harvard University Press.

Monbiot, G. (1 de mayo, 2016). Neoliberalismo: la raíz ideológica de todos nuestros problemas. El Diario. Recuperado de https://bit.ly/1QMkqmP

Reig, R. (2014). La investigación dependiente: crítica estructural al sistema JCR. Ámbitos. Revista Internacional de Comunicación, 27, 1-36.

Rivero, R. (2013). Educación y Pedagogía en el marco del neoliberalismo y la globalización. Perfiles Educativos, 142, $149-166$.

Rizvi, F. y Lingard, B. (2009) Globalizing educational policy. London: Routledge.

Saura, G. y Luengo, J. (2015). Biopolítica y educación. Medición, estandarización, regulación poblacional. Teor. educ. 27(2), 115-135. doi: http://dx.doi.org/10.14201/teoredu2015272115135

Silva, A. (1976). Paulo Freire: una educación para la liberación. Revista de Educación, 242, 87-96.

Vallaeys, F. (2014). La responsabilidad social universitaria: un nuevo modelo universitario contra la mercantilización. Universia, 12(5), 105-117. Recuperado de: https://ries.universia.net/article/download/137/18

\section{BY-NC-ND}

